\title{
Phorbol Esters Target the Activity-Dependent Recycling Pool and Spare Spontaneous Vesicle Recycling
}

\author{
Tuhin Virmani, ${ }^{1}$ Mert Ertunc, ${ }^{1}$ Yildirim Sara, ${ }^{3}$ Marina Mozhayeva, ${ }^{1}$ and Ege T. Kavalali ${ }^{1,2}$ \\ ${ }^{1}$ Center for Basic Neuroscience and ${ }^{2}$ Department of Physiology, University of Texas Southwestern Medical Center, Dallas, Texas 75390-9111, and \\ ${ }^{3}$ Department of Pharmacology, Hacettepe University Faculty of Medicine, Sihhiye 06100, Ankara, Turkey
}

Using electrophysiology and styryl dye imaging, we studied the effect of phorbol 12-myristate 13-acetate (PMA) on activity-dependent and spontaneous vesicle recycling. In electrophysiological experiments, we found that the PMA effect depended on the maturational state of the synapses. Spontaneous neurotransmitter release from nascent synapses without a functional readily releasable pool (RRP) was unresponsive to PMA application. In contrast, mature synapses responded robustly to PMA application, consistent with previous studies. Using styryl dye imaging, we found that there was a PMA-dependent increase in the size of the RRP when PMA was present before, during, or after activity-dependent dye uptake, suggesting that this effect involves an increase in the population of the RRP by vesicles recruited from the reserve pool. Additionally, we found that when PMA was present during spontaneous dye uptake, there was an increase in dye labeling, and these additional dye-loaded vesicles showed rapid destaining in response to strong stimulation and were also releasable by hypertonic sucrose. In contrast, these observations were not reproducible when PMA treatment was performed after spontaneous dye uptake and extracellular dye washout. Together, these findings suggest that the increased spontaneous neurotransmission in the presence of PMA was attributable to release of vesicles from the RRP rather than an effect of PMA on the spontaneously recycling pool. Thus, the phorbol esters selectively regulate the activity-dependent pool of vesicles, indicating that priming mechanisms that prepare vesicles for fusion, which are targeted by phorbol esters, are different for the spontaneous and evoked forms of fusion.

Key words: synaptic vesicle recycling; spontaneous fusion; FM2-10; phorbol esters; neurotransmission; readily releasable pool

\section{Introduction}

The synchronous release of neurotransmitter from synaptic vesicles is a very precisely regulated process. Diacylglycerol (DAG) is a key signaling molecule involved in this regulation. Under normal conditions, plasma membranes contain very little DAG. However, activation of $\mathrm{G}_{\mathrm{q}}$-coupled receptors stimulates phospholipase $\mathrm{C} \beta$, which catalyzes the cleavage of phosphatidylinositol 4,5-bisphosphate into the small signaling molecules DAG and inositol 1,4,5-trisphosphate. Activation of protein kinase C (PKC) is thought to be the classical target of DAG action in the synapse. However, recent evidence suggests that the synaptic protein munc-13 may also serve as a major target (Rhee et al., 2002).

Experiments using the DAG analog phorbol esters have shown a positive regulation of synaptic transmission (Malenka et al., 1986; Shapira et al., 1987). Several lines of evidence suggest that this regulation is mediated through increases in the size of the readily releasable pool (RRP), as well as $\mathrm{Ca}^{2+}$ affinity of the fusion machinery. In central synapses, this result has been shown electrophysiologically, optically using styryl dyes, and by bio-

\footnotetext{
Received May 11, 2005; revised 0ct. 7, 2005; accepted 0ct. 8, 2005.

This work was supported by the National Institute of Mental Health Grant MH068437 (E.T.K.). We thank F. Deák and $C$. Wasser for critically reading this manuscript.

Correspondence should be addressed to Dr. Ege T. Kavalali, Center for Basic Neuroscience, University of Texas Southwestern Medical Center, 5323 Harry Hines Boulevard, Dallas, TX 75390-9111. E-mail: Ege.Kavalali@ UTSouthwestern.edu.

DOI:10.1523/JNEUROSCI.3766-05.2005

Copyright $\odot 2005$ Society for Neuroscience $\quad$ 0270-6474/05/2510922-08\$15.00/0
}

chemistry (Stevens and Sullivan, 1998; Lonart and Südhof, 2000; Waters and Smith, 2000). Similar effects of phorbol esters have also been obtained in multiple different systems including chromaffin cells (Yang et al., 2002), retinal bipolar cells (Berglund et al., 2002), spinal dorsal horn neurons (Robinson and Zhuo, 2004), calyces of Held ( $\mathrm{Wu}$ and $\mathrm{Wu}, 2001$ ), and frog neuromuscular junctions (Angleson and Betz, 2001). Phorbol esters can also cause an increase in the frequency of miniature postsynaptic currents (Shapira et al., 1987; Angleson and Betz, 2001; Rhee et al., 2002). This increase in miniature events may be dependent on activation of L-type calcium channels and, thus, may be independent of phorbol ester action on the RRP (Waters and Smith, 2000). Recent results suggest that spontaneous vesicles recycle independently of the activity-dependent recycling vesicle pool (Sara et al., 2005). Together, these previous findings imply a potential link between the segregation of vesicle pools and phorbol ester regulation.

To examine whether phorbol esters are specific regulators of a select set of vesicles within a synapse, we studied the effect of the phorbol ester phorbol 12-myristate 13-acetate (PMA) on activity-dependent and spontaneous vesicle recycling using whole-cell recordings as well as the styryl dye FM2-10. We found that PMA selectively acts on the activity-dependent recycling pool in mature synapses by mobilizing reserve pool vesicles into the RRP. Our results suggest that the increase in miniature events observed during or after PMA application is attributable to re- 
lease from the readily releasable pool rather than mobilization of spontaneously recycling vesicles.

\section{Materials and Methods}

Cell culture. Dissociated hippocampal cultures were prepared from 2- to 3-d-old Sprague Dawley rat pups using methods described previously (Mozhayeva et al., 2002). Experiments were performed after 14-21 d in vitro (DIV), corresponding to a time period when synapses reach full maturity in culture (except in experiments presented in Fig. 1).

Electrophysiology. Pyramidal cells were voltage clamped to $-70 \mathrm{mV}$ using whole-cell patch-clamp technique. The electrode solution contained the following (in mM): $115 \mathrm{Cs}-\mathrm{MeSO}_{3}, 10 \mathrm{CsCl}, 5 \mathrm{NaCl}, 10$ HEPES, 0.6 EGTA, 20 tetraethylammonium chloride, 4 Mg-ATP, 0.3 $\mathrm{Na}_{2}$ GTP, 10 QX-314 (lidocaine $N$-ethyl bromide), pH 7.35, 300 mOsm (Sigma, St. Louis, MO). A modified Tyrode solution was the extracellular solution used in all experiments (including fluorescence imaging), and it contained the following (in mM): $150 \mathrm{NaCl}, 4 \mathrm{KCl}, 2 \mathrm{MgCl}_{2}, 10$ glucose, 10 HEPES, and $2 \mathrm{CaCl}_{2}, \mathrm{pH} 7.4, \sim 310 \mathrm{mOsm}$. Data was acquired using an Axopatch 200B amplifier and Clampex 8.0 software (Molecular Devices, Sunnyvale, CA). Recordings were filtered at $2 \mathrm{kHz}$ and sampled at $5 \mathrm{kHz}$. Spontaneous events were recorded in the presence of $1 \mu \mathrm{M}$ tetrodotoxin (TTX). For measuring evoked responses, electrical stimulation was delivered through parallel platinum electrodes in modified Tyrode solution (see below) without CNQX or AP-5.

PMA (Sigma) was applied at a concentration of $1 \mu \mathrm{M}$. For the folimycin-treatment experiments, a single coverslip was treated in the modified Tyrode solution containing $67 \mathrm{~nm}$ folimycin (Calbiochem, La Jolla, CA) dissolved in DMSO (Sigma) or vehicle alone for $10 \mathrm{~min}$ in the presence of $1 \mu \mathrm{M}$ TTX. A single cell was held in the whole-cell recording configuration during the treatment, and evoked responses were tested before and after the treatment (Sara et al., 2005). All statistical comparisons were performed with the two-tailed unpaired $t$ test, and values are given as mean \pm SEM.

Fluorescence imaging. Synaptic boutons were loaded with FM2-10 (400 $\mu \mathrm{M}$; Invitrogen, Eugene, OR) under conditions outlined in the text. Ninety second incubation in $47 \mathrm{~mm} \mathrm{~K}^{+}$solution [Tyrode solution with equimolar substitution of $\mathrm{KCl}(47 \mathrm{~mm}$ for $\mathrm{NaCl})$ ] gives maximal labeling of the recycling vesicle pool in a given synapse (Harata et al., 2001). The $47 \mathrm{~mm} \mathrm{~K}^{+}$solution is obtained by rapid mixing of equal volumes $(150 \mu \mathrm{l}$ each) of dye-free $4 \mathrm{~mm} \mathrm{~K}^{+}$and dye-containing $90 \mathrm{~mm} \mathrm{~K}^{+}$solutions in the imaging chamber. This procedure does not necessitate dye perfusion through tubing, which in our hands results in build-up nonspecific background staining. Spontaneous loadings were performed in modified Tyrode solution containing $1 \mu \mathrm{M}$ TTX to inhibit action potentials induced by network activity inherent in the culture. Phorbol ester treatments were performed at a final concentration of $1 \mu \mathrm{M}$ PMA or $4 \alpha$-PMA (Sigma). Images were taken after $10 \mathrm{~min}$ washes in dye-free solution with nominal $\mathrm{Ca}^{2+}$ to minimize spontaneous dye loss. Destaining of hippocampal terminals with high-potassium challenge was achieved by direct perfusion of solutions onto the field of interest by gravity $(2 \mathrm{ml} / \mathrm{min})$. In a typical experiment, high potassium challenge was applied at least three times (for $90 \mathrm{~s}$ each separated by $60 \mathrm{~s}$ intervals) to release all of the dye trapped in presynaptic terminals (supplemental Fig. 1, available at www.jneurosci.org as supplemental material). All activity-dependent and sucrose staining and destaining protocols were performed in the presence of $10 \mu \mathrm{M} C N Q X$ and $50 \mu \mathrm{M}$ AP-5 to prevent recurrent activity. In all experiments, we selected isolated boutons $\left(\sim 1 \mu \mathrm{m}^{2}\right)$ for analysis and avoided apparent synaptic clusters. Images were obtained using a cooled, intensified digital CCD camera (Roper Scientific, Trenton, NJ) during illumination $(1 \mathrm{~Hz} ; 40 \mathrm{~ms})$ at $480 \pm 20 \mathrm{~nm}$ (505 dichroic long pass; $535 \pm 25$ band pass) via an optical switch (Sutter Instruments, Novato, CA). Images were acquired and analyzed using Axon Imaging Workbench software (Molecular Devices). All statistical analyses were performed using Student's two-tailed $t$ test, using the number of coverslips (derived from at least two independent cultures) as $n$ unless stated otherwise. Experimental results are represented as mean \pm SEM.

\section{Results}

PMA-induced augmentation of spontaneous fusion frequency depends on the maturational stage of synapses

In a previous study in dissociated hippocampal cultures, we found that immediately after synapse formation ( $\sim 5 \mathrm{DIV})$, a substantial portion of the presynaptic terminals did not respond

\section{mEPSCs DIV5}

A

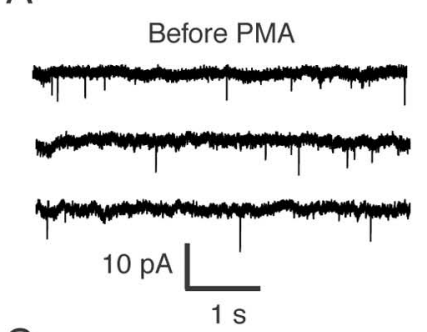

C

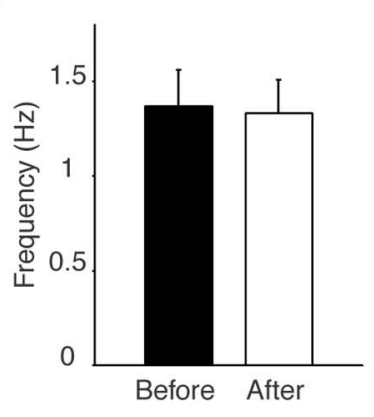

B

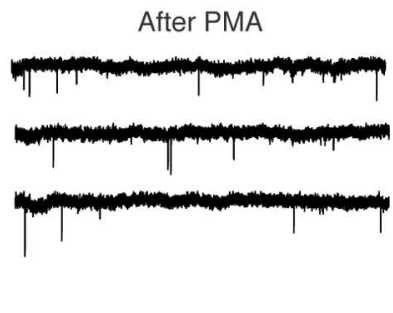

D

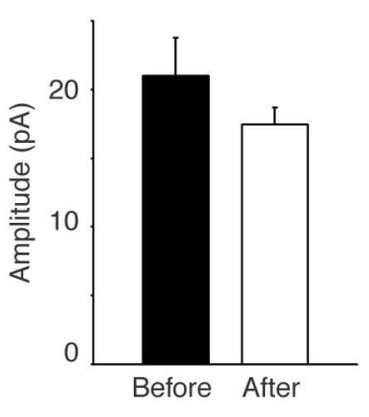

mEPSCs DIV15

$E$

F

Before PMA
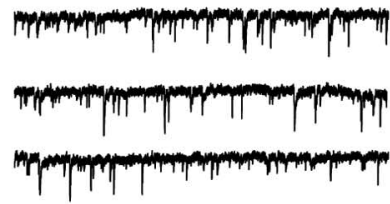

$20 \mathrm{pAL}$

$500 \mathrm{~ms}$

$G$

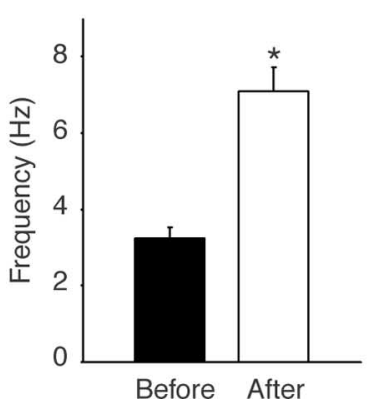

$\mathrm{H}$

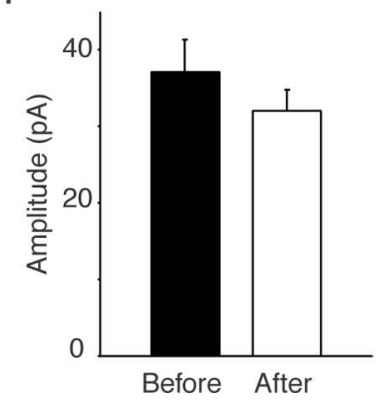

Figure 1. PMA application increased the frequency of miniature EPSCs (mEPSCs) in 15 DIV hippocampal cultures but not in 5 DIV cultures. Recordings were made before and 2 min after PMA application. $A, B$, Sample traces showing the mEPSCs before and after PMA application at 5 DIV. C, D, Frequency and amplitude of mEPSCs did not change after PMA treatment in 5 DIV hippocampal cultures $(n=7) . \boldsymbol{E}, \boldsymbol{F}$, Sample traces of whole-cell patch-clamp recordings showing mEPSCs obtained from neurons in 15 DIV hippocampal cultures. G, Two minute application of PMA increased the mEPSC frequency ( $n=7$ for each group). ${ }^{*} p<0.001$. $\boldsymbol{H}$, Amplitudes of $\mathrm{mEPSCs}$ did not change after $2 \mathrm{~min}$ of PMA treatment. Error bars indicate SE. 

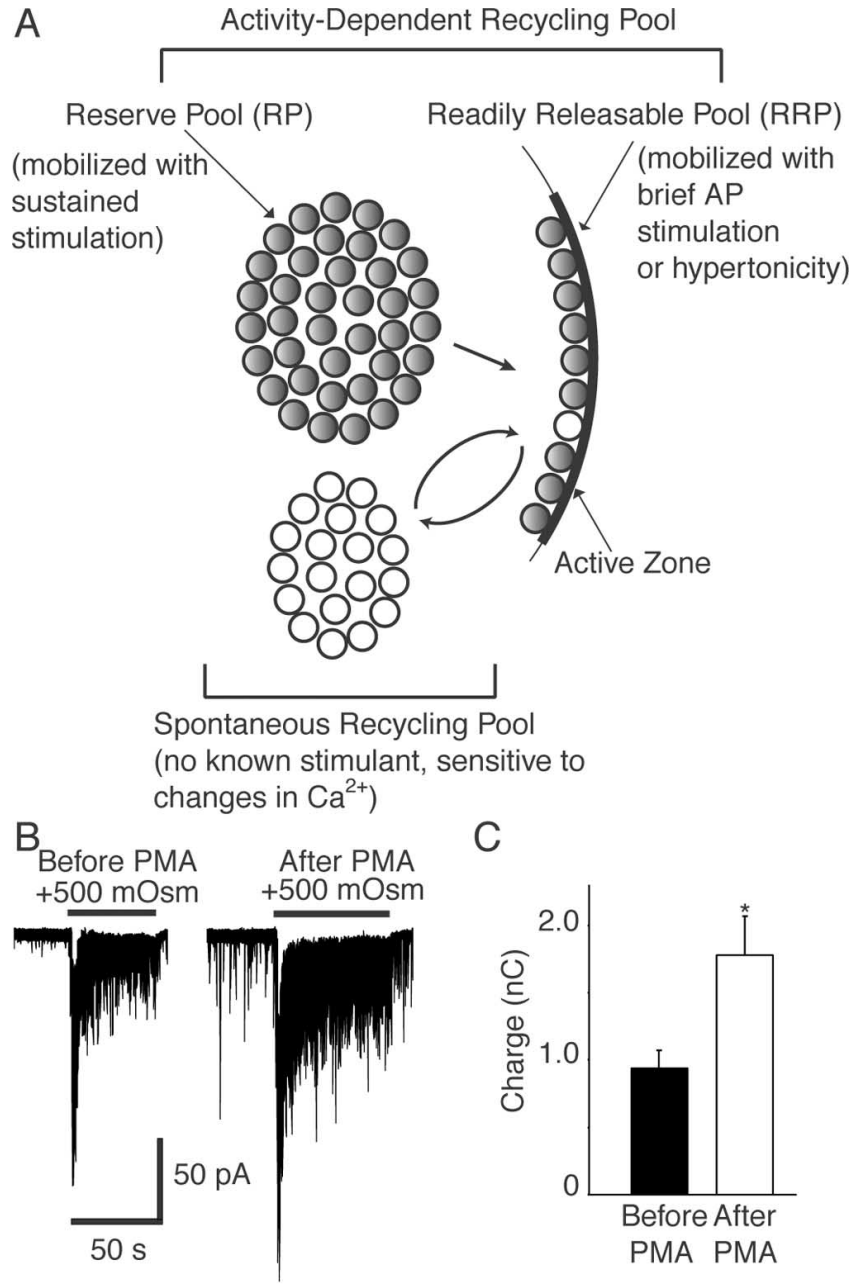

D
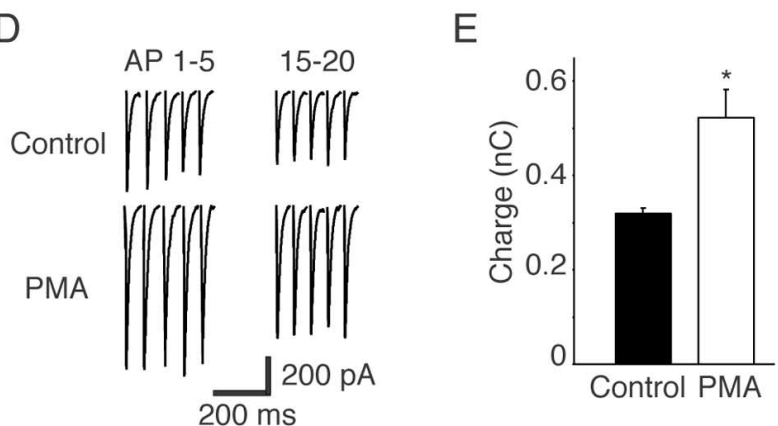

Figure 2. A, Studies in several secretory preparations and central neuronal synapses have classified synaptic vesicles with respect to their relative availability for release during stimulation. The vesicles in the RRP are immediately available for release after arrival of an action potential. RRP vesicles can be mobilized by a brief burst of action potentials or by application of hypertonic solutions (Rosenmund and Stevens, 1996). A reserve pool (RP) of vesicles is spatially distant from the release sites and replaces the vesicles in the RRP that have undergone exocytosis. RP vesicles can typically be mobilized by sustained trains of action potentials or by elevated $\mathrm{K}^{+}$stimulation. Together, the RRP and reserve pool make up the recycling pool of vesicles, which corresponds to all vesicles capable of activity-dependent recycling (all gray vesicles) after stimulation. Recent evidence suggests that spontaneous neurotransmission is driven by a set of vesicles recycling independently of the activity-dependent vesicle pool (white vesicles) (Sara et al., 2005). Note that a comparison of the total number of vesicles measurable in synapses through physiological means versus morphological analysis supports the presence of a number of vesicles that are not mobilized by activity under standard paradigms. The mechanisms that would fully mobilize this "resting" pool of vesicles (not depicted in the figure) remain to be determined. $\boldsymbol{B}$, PMA application increased the size of the RRP in 15 DIV hippocampal cultures. Sample whole-cell patch-clamp recordings of synaptic responses to the application of $500 \mathrm{~mm}$ sucrose solution before and $2 \mathrm{~min}$ after PMA treatment are shown. C, Plot showing an increase in the size of sucrose response after PMA treatment, measured as the total charge $(n=$
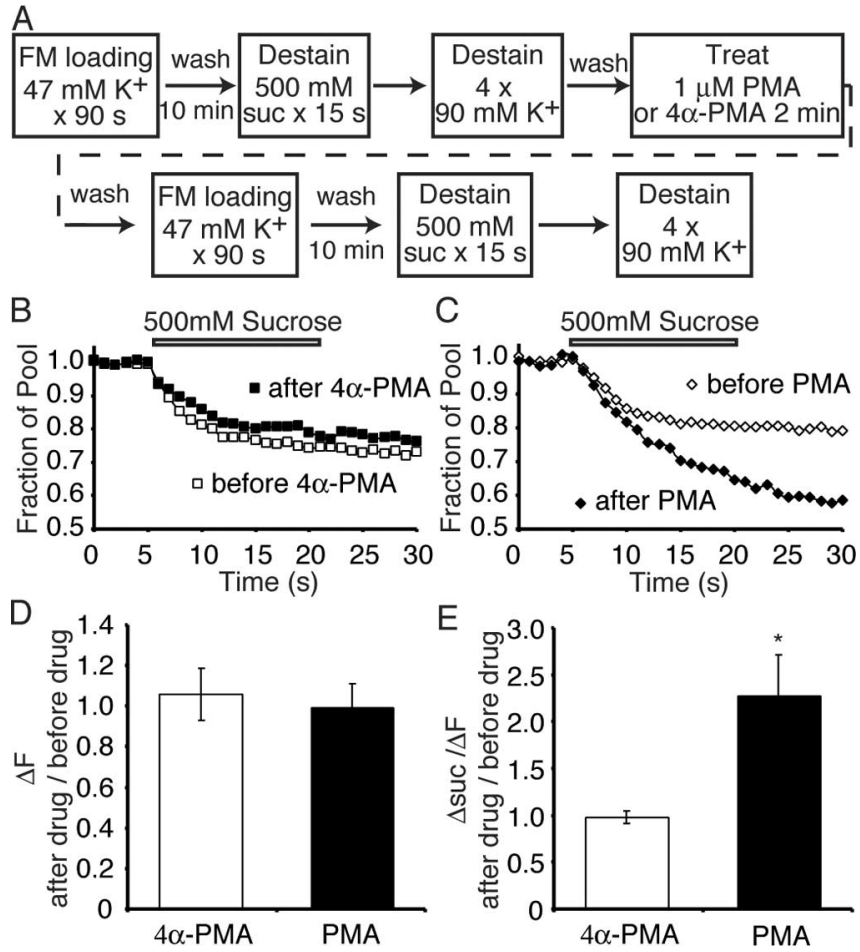

Figure 3. PMA increases the size of the RRP detected optically. $A$, Synapses were loaded with FM2-10 by depolarization with a $47 \mathrm{~mm} \mathrm{~K}^{+}$solution for $90 \mathrm{~s}$. After dye washout, the synapses were imaged during a $15 \mathrm{~s}$ sucrose application followed by multiple rounds of $90 \mathrm{~mm} \mathrm{~K}$ application. After a 5 min wash, the same synapses were treated with $1 \mu \mathrm{M}$ PMA or $4 \alpha$-PMA for $2 \mathrm{~min}$. After another $5 \mathrm{~min}$ wash to remove residual PMA, these synapses were subjected to a second round of $47 \mathrm{~mm} \mathrm{~K}{ }^{+}$loading and subsequent destaining. B, C, Sample experimental traces showing the average sucrose response of 110 synapses from a single coverslip before and after $4 \alpha$-PMA (B) or PMA $(\boldsymbol{C})$ application. $\boldsymbol{D}$, The average ratio of the total recycling pool in synapses after treatment to that before treatment. $\boldsymbol{E}$, The average ratio of $\Delta \mathrm{suc} / \Delta F$ in synapses after treatment to that before treatment shows a significant increase after PMA treatment compared with $4 \alpha$-PMA treatment ( $n=3$ and 6 coverslips for $4 \alpha$-PMA and PMA, respectively, with $\sim 100$ synapses imaged per coverslip). ${ }^{*} p<0.05$. Error bars indicate SE.

to brief action potential stimulation or hypertonic sucrose application, although they released neurotransmitter spontaneously or in response to strong elevated $\mathrm{K}^{+}$-induced depolarization (Mozhayeva et al., 2002). Together with the ultrastructural observation that in these young cultures a majority of synapses lacked a docked cluster of synaptic vesicles at the surface membrane, we had arrived at the conclusion that these nascent synapses lacked an RRP of vesicles. Here, we used this setting to test whether the well established effect of PMA on spontaneous fusion frequency required formation of an RRP. Interestingly, application of $1 \mu \mathrm{M}$ PMA on 5 DIV hippocampal cultures did not augment spontaneous fusion frequency in the presence of TTX (Fig. 1A-D). In contrast, the same PMA application caused a twofold to threefold increase in spontaneous fusion frequency in mature cultures (15 DIV) (Fig. $1 E-H$ ). This developmental specificity of the PMA action is consistent with the premise that the

$\leftarrow$

3). ${ }^{*} p<0.05 . D$, Sample traces of whole-cell patch-clamp recordings showing the first five and last five responses to a $20 \mathrm{~Hz}, 2 \mathrm{~s}$ train of action potentials recorded before and after application of PMA in 15 DIV neuronal cell cultures. $\boldsymbol{E}$, Plot showing the PMA-dependent increase in total synaptic charge transfer in response to $20 \mathrm{~Hz}, 2$ s electrical stimulation compared with controls $(n=5) .{ }^{*} p<0.05$. Error bars indicate SE. 


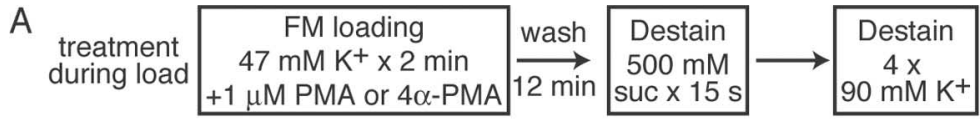

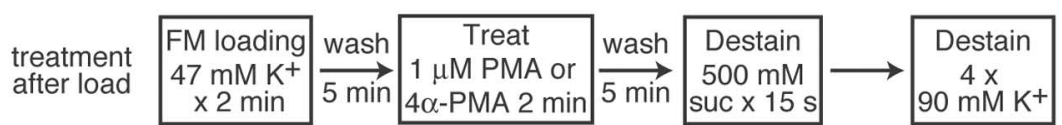
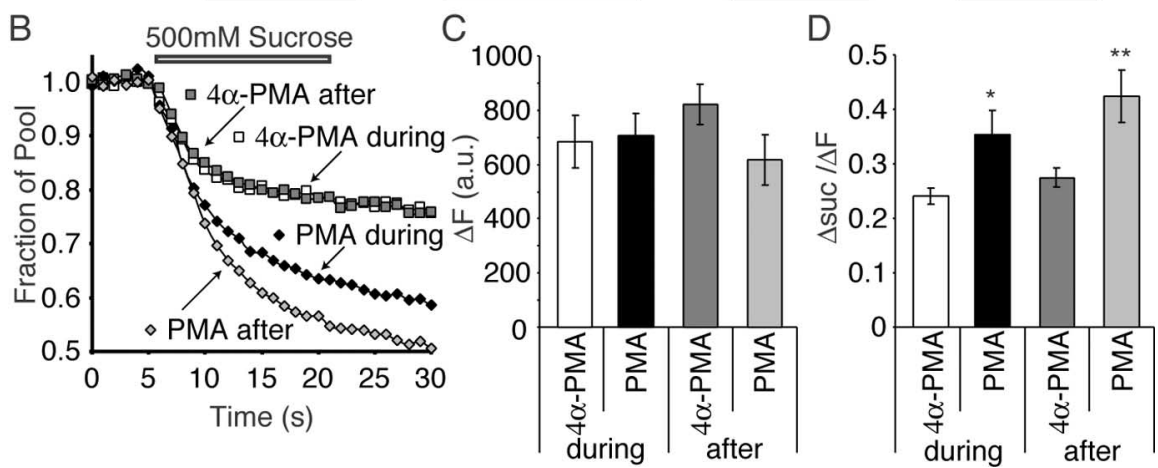

Figure 4. Activity is not necessary during PMA treatment for redistribution of vesicles between pools. $\boldsymbol{A}$, Separate sets of synapses were treated with PMA or $4 \alpha$-PMA either during loading with $47 \mathrm{mM} \mathrm{K}^{+}$or after $5 \mathrm{~min}$ of dye washout. After washout of dye and residual PMA for an additional 5 min, synapses were imaged during a 15 s application of 500 mm sucrose, followed by multiple rounds of $90 \mathrm{~mm} \mathrm{~K}^{+}$solution for maximal destaining. $\boldsymbol{B}$, Sample responses of synapses from a single coverslip during $15 \mathrm{~s}$ of $500 \mathrm{~mm}$ sucrose application. Synapses treated with PMA showed faster kinetics of dye loss compared with synapses treated with $4 \alpha$-PMA as a control. $C$, Total vesicle pool size as measured by the dye fluorescence trapped in synapses $(\Delta F)$ was not significantly different between the different loading paradigms ( $n=6-7$ coverslips each), although there was a tendency toward a smaller pool size in cultures treated with PMA after dye loading and washout compared with $4 \alpha$-PMA, suggesting some spontaneous dye loss after PMA treatment (PMA treatment after load, $75 \pm 11 \%$ of $4 \alpha$-PMA-treated synapses; $p=0.12$ ). $D$, The ratio of dye loss during sucrose application to the total pool size, labeled $\Delta \mathrm{suc} / \Delta F$, showed a significant increase in synapses treated with PMA either during or after dye loading ( $n=6-7$ coverslips each). ${ }^{*} p<0.05 ;{ }^{* *} p<0.02$. Error bars indicate SE.

PMA effect is specific to a set of vesicles resident in the RRP and does not affect vesicle fusion in general.

Next, we tested whether the PMA effect enhanced the size of the RRP in mature cultures. To probe the RRP, we triggered neurotransmitter release by local application of hypertonic sucrose or by 40 action potentials applied at $20 \mathrm{~Hz}$. Indeed, as seen in previous studies (Stevens and Sullivan, 1998), both forms of neurotransmitter release responded robustly to the application of PMA (Fig. 2). The extent of release was augmented twofold in 15 DIV cultures (Fig. $2 B, D$ ). In contrast, hypertonic sucrose perfusion did not induce significant neurotransmitter release in $5 \mathrm{DIV}$ cultures, replicating our previous observations (Mozhayeva et al., 2002). Additionally, this release was not modified by PMA application (data not shown).

\section{PMA increases the size of the RRP without any change in the total pool}

What is the origin of vesicles that augment the RRP size after PMA application? To address this question, we loaded synapses with FM2-10 dye using $47 \mathrm{~mm} \mathrm{~K}^{+} / 2 \mathrm{mM} \mathrm{Ca}^{2+}$ application for $90 \mathrm{~s}$ (Fig. 3A). After a $10 \mathrm{~min}$ washout period, we imaged synapses during a $15 \mathrm{~s}$ application of $500 \mathrm{~mm}$ sucrose solution to measure the size of the RRP (Rosenmund and Stevens, 1996). We then continued to image during maximal destaining achieved by application of multiple rounds of $90 \mathrm{~mm} \mathrm{~K}^{+}$solution. This complete destaining provided us with the total recycling vesicle pool size in these synapses (supplemental Fig. 1, available at www. jneurosci.org as supplemental material). After a brief 5 min wash, the synapses were treated with either $1 \mu \mathrm{M}$ PMA or $4 \alpha$-PMA for 2 min and then washed again for 5 min to remove any residual PMA or $4 \alpha$-PMA. We then performed a second round of $47 \mathrm{~mm}$
$\mathrm{K}^{+}$loading and destaining, similar to that described above to determine what effect PMA has on this vesicle pool.

Under these conditions, the treatment of synapses with PMA increased sucrosedependent dye release (averaged sample traces before and after PMA) (Fig. 3B,C) (supplemental Fig. 1, available at www. jneurosci.org as supplemental material) as well as increased the ratio of the dye released by $15 \mathrm{~s}$ of sucrose to the total pool $(\Delta \mathrm{suc} / \Delta F)$ (Fig. 3E). Neither of these parameters was altered by treatment with the inactive form of PMA, $4 \alpha$-PMA (Fig. $3 B, D, E)$. This increase in the ratio of RRP to total pool $(\Delta \mathrm{suc} / \Delta F)$ observed after PMA application is also consistent with the twofold increase in the sucrose response measured electrophysiologically after PMA application (Fig. $2 B$ ).

Comparing the ratio of $\Delta F$ after drug treatment versus before treatment did not reveal any significant change with PMA application compared with $4 \alpha$-PMA (Fig. $3 D)$. However, when we plotted $\Delta$ suc/ $\Delta F$ obtained after PMA treatment versus before PMA treatment, there was a twofold increase in the size of the RRP after PMA treatment compared with $4 \alpha$-PMA controls (Fig. 3E). This would suggest that PMA does not increase the total vesicle pool size, but reconfigures the distribution of vesicles between the RRP and the reserve pool.

\section{PMA redistributes vesicles from the reserve pool to the RRP regardless of activity during treatment}

To further explore the increase in the RRP size, we used two different loading protocols on two populations of synapses. In one set, we loaded synapses with $47 \mathrm{mM} \mathrm{K}^{+}$in the presence of either PMA or $4 \alpha$-PMA. In a second set of experiments, we loaded synapses with $47 \mathrm{mM} \mathrm{K}^{+}$and treated them with either PMA or $4 \alpha$-PMA after washout of extracellular dye (Fig. $4 A$ ). Similar to treatment before dye loading, synapses that were loaded by activity in the presence of PMA had an increased sucrose-dependent dye loss compared with $4 \alpha$-PMA control synapses (Fig. $4 B$ ). Somewhat surprisingly, yet not completely unexpectedly, synapses treated with PMA after dye loading and washout also showed an increased rate of sucrose-dependent dye release (Fig. $4 B$ ). When we plotted the average median size of the total recycling pool in these four conditions, there was no significant change (Fig. 4C). However, there was a significant increase in the ratio of dye released by $15 \mathrm{~s}$ sucrose application either when PMA was applied during the dye loading stage or after dye loading and extracellular washout, compared with two conditions of $4 \alpha$-PMA treatment (Fig. 4D). Given the fact that the PMA application increases the kinetics and amount of sucrose-dependent dye loss from vesicles loaded by activity, regardless of the application during or 5 min after loading, this finding further suggests that PMA-dependent cascades are shuttling vesicles from the reserve pool into the RRP without increasing the total number of vesicles that can recycle. 


\section{PMA does not affect spontaneous vesicle recycling}

Recent evidence suggests that the spontaneous neurotransmission is driven by a set of vesicles recycling independently of the activity-dependent vesicle pool (Sara et al., 2005). Therefore, we next tested whether PMA had any effect on the spontaneous synaptic vesicle recycling. To this end, we loaded spontaneously recycling vesicles with FM2-10 dye for 10 min using a solution of 4 $\mathrm{mM} \mathrm{K} \mathrm{K}^{+} / 2 \mathrm{mM} \mathrm{Ca}^{2+}$ in the presence of $1 \mu \mathrm{M}$ TTX to inhibit activity. Once again, we either treated the synapses with PMA or $4 \alpha$-PMA during the dye-loading period or for $10 \mathrm{~min}$ after the loading protocol, when extracellular dye had been removed. We then imaged the synapses during multiple rounds of destaining with $90 \mathrm{mM} \mathrm{K}^{+}$(Fig. 5A).

When we plotted the total dye uptake during the different loading conditions, we observed that there was a selective increase in the amount of dye uptake when PMA was present during the FM dye loading (Fig. 5B). This was coupled with an increased rate of dye release and the development of biexponential release kinetics in response to $90 \mathrm{mM} \mathrm{K}^{+}$(Fig. 5C). Treatment with PMA after dye loading and washout did not show any change in pool size, and the release kinetics showed normal slow monoexponential activity-dependent release similar to $4 \alpha$-PMA control treatments. When we fit the destaining patterns after these various loading conditions with two exponential curves, we found that there was a selective decrease in the time constant of the fast component of release in synapses treated with PMA during the loading (Fig. 5D), without any change in the slow time constant (Fig. 5E). Additionally, the ratio of the proportion of the fast component to the slow component is significantly increased in synapses treated with PMA during loading (Fig. $5 F$ ). This increase in fast-release kinetics would suggest that some dye-loaded vesicles are resident in the activity-dependent recycling pool.

\section{PMA-dependent increase in pool size in the absence of activity is attributable to the labeling of vesicles that normally populate the RRP}

When synapses were loaded using activity, PMA treatment during or after loading had a similar effect on the RRP size (Fig. 4). However, PMA treatment during and after spontaneous loading did not lead to parallel effects. The increase in the pool size loaded in addition to the biexponential release kinetics suggests that when PMA is present during loading, it can mobilize vesicles that populate the RRP.

To test this possibility further, we challenged spontaneously labeled vesicles with hypertonic sucrose application. We loaded FM2-10 into vesicles in the presence of TTX. PMA or $4 \alpha$-PMA was present either during the $10 \mathrm{~min}$ of dye application or $5 \mathrm{~min}$ after dye washout. After dye washout, synapses were first imaged during a $15 \mathrm{~s}$ pulse of sucrose to release the RRP, followed by maximal destaining using multiple rounds of high $\mathrm{K}^{+}$stimulation (Fig. 6A). We found that, when PMA was present during loading, there was a component of loaded dye that was releasable by sucrose, in contrast to the minimal dye loss on sucrose application of $4 \alpha$-PMA-treated synapses (Fig. $6 B$ ). When PMA or $4 \alpha$-PMA was applied after dye washout, there was also minimal sucrose release. The absolute amount of dye released by sucrose (Fig. 6C), as well as the fraction of the dye-loaded pool (Fig. 6D), was significantly larger in synapses treated with PMA during loading compared with all of the other conditions. The amount of dye released by sucrose $[\Delta F=35 \pm 3$ arbitrary units (a.u.) ] from synapses treated with PMA during spontaneous loading was approximately one-half of the increased dye uptake (increase in $\Delta F$ relative to control $=65 \pm 8$ a.u.) during the same loading proto-
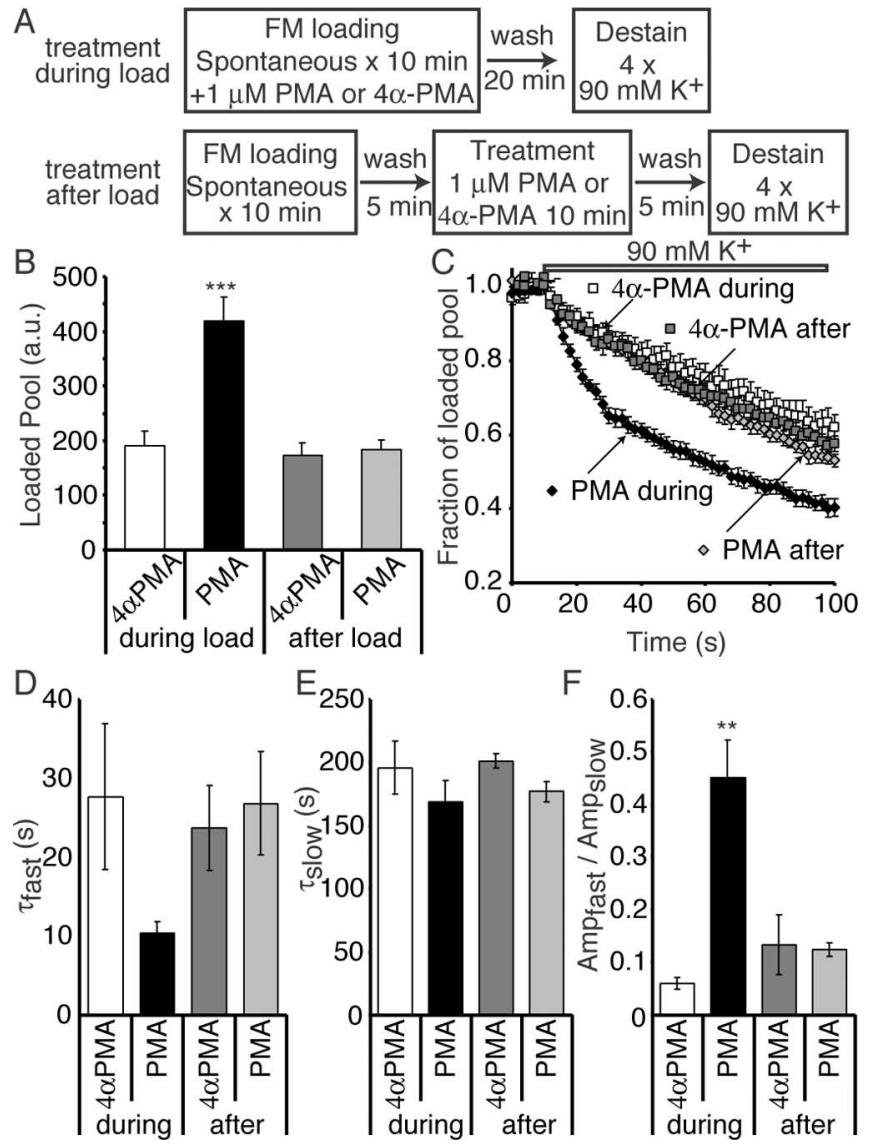

Figure 5. Increased dye labeling and faster activity-dependent destaining kinetics after PMA treatment during spontaneous dye uptake. $A$, Synapses were treated with $1 \mu \mathrm{m}$ PMA or $4 \alpha$-PMA either during spontaneous FM2-10 dye loading (in $4 \mathrm{~mm} \mathrm{~K}^{+} / 2 \mathrm{~mm} \mathrm{Ca}^{2+}$ and $1 \mu \mathrm{M}$ TTX) or after $5 \mathrm{~min}$ of dye washout for $10 \mathrm{~min}$. After an additional $5 \mathrm{~min}$ of dye washout, synapses were imaged during multiple rounds of $90 \mathrm{~mm} \mathrm{~K}^{+}$application. $\boldsymbol{B}$, Plot showing that the average median size of the dye-labeled pool increases when PMA is present during dye loading ( $n=5-9$ coverslips for each condition). ${ }^{* * *} p<0.001$ for PMA during dye load compared with all other loading conditions. C, Plot showing the average kinetics of dye destaining in response to high $\mathrm{K}^{+}$application in different loading conditions. Dye destaining in synapses in which PMA was present during the loading protocol show biphasic release typical of dye release from the evoked pool. Dye release in all other conditions shows monoexponential slow dye loss typical of high $\mathrm{K}^{+}$destaining of the spontaneous vesicle pool. $\boldsymbol{D}$, The fast time constant of release in a double-exponential fit of dye release under $90 \mathrm{~mm} \mathrm{~K}^{+}$application is faster in synapses loaded in the presence of PMA compared with other loading paradigms. Here, we should note that double-exponential fits were used for all traces to facilitate quantification and comparison. Traces from individual experiments could be more reliably described with two exponentials (albeit slow ones for all conditions except for "PMA during"), although the average traces shown in C (again except for "PMA during") may not seem to justify double-exponential fits. $\boldsymbol{E}$, The slow time constant in a double-exponential fit of the release kinetics was not altered by PMA. $\boldsymbol{F}$, The ratio of the amplitude of the fast-to-slow component of dye release was significantly larger in synapses that were loaded in the presence of PMA. ${ }^{* *} p<0.005$ between PMA during load and all other loading conditions. Error bars indicate $S E$.

col. This is consistent with mixing of these vesicles with the reserve pool. These findings suggest that the additional loading observed during PMA treatment is attributable to a PMAdependent release of vesicles that would normally release under activity, and these vesicles then populate the normal activitydependent recycling pool and show biphasic release kinetics. Alternatively, PMA could shuttle spontaneously endocytosing vesicles into the RRP when present during loading. We consider the latter possibility unlikely, because the increase in the labeled pool size after PMA treatment nicely corresponds to 
A
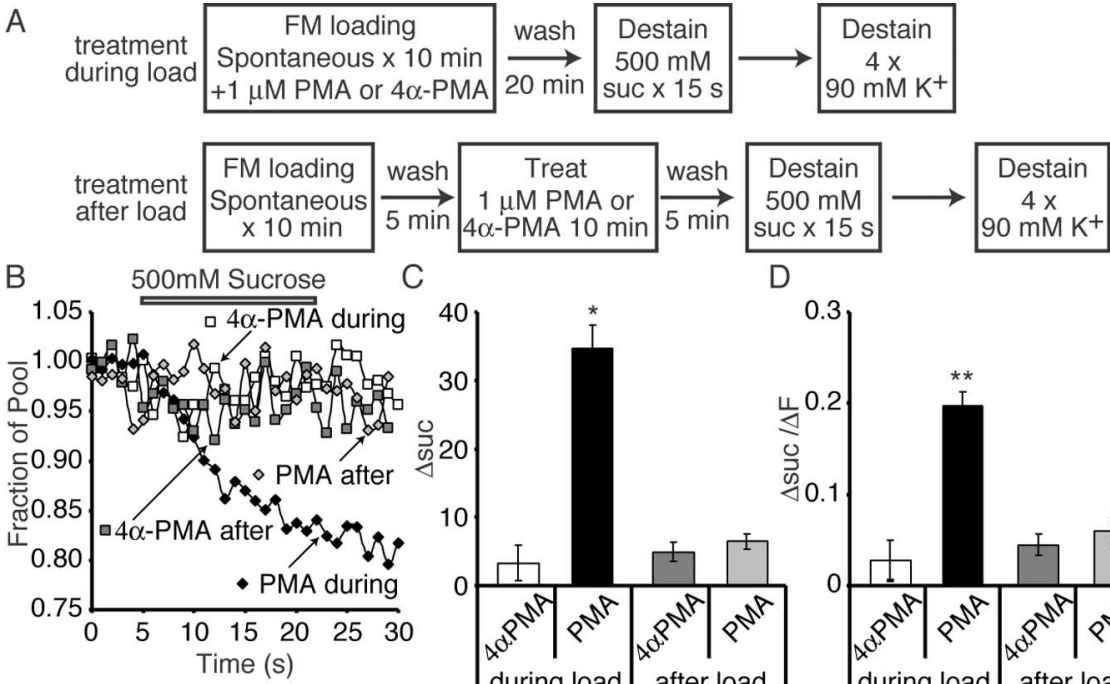

C

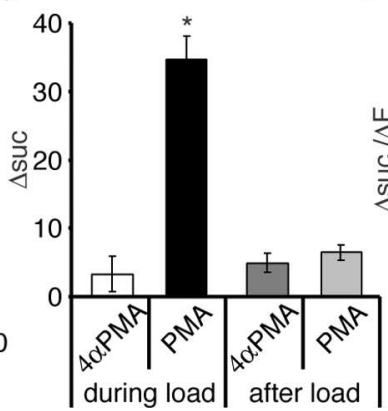

$\mathrm{D}$

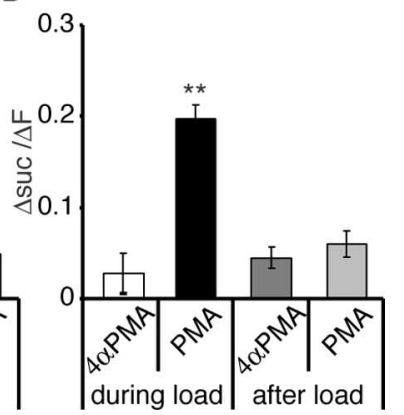

Figure 6. The population of vesicles mobilized by PMA application in the presence of TTX populate the RRP. A, Synapses were treated with $1 \mu \mathrm{M}$ PMA or $4 \alpha$-PMA either during spontaneous FM2-10 dye loading (in $4 \mathrm{~mm} \mathrm{~K}^{+} / 2 \mathrm{mM} \mathrm{Ca}^{2+}$ and $1 \mu \mathrm{M} \mathrm{TTX)} \mathrm{or} \mathrm{after}$ $5 \mathrm{~min}$ of dye washout for $10 \mathrm{~min}$. After an additional $5 \mathrm{~min}$ of dye washout, synapses were imaged during multiple rounds of $90 \mathrm{~mm}$ $\mathrm{K}^{+}$application. B, Sample traces of dye release during $15 \mathrm{~s}$ of sucrose application. Synapses loaded in the presence of PMA show faster dye release than $4 \alpha$-PMA-treated synapses, which show minimal sucrose release. C, Plotting the absolute dye loss during sucrose, we see a significant increase in synapses treated with PMA during loading ( $n=3$ coverslips each). ${ }^{*} p<0.01$. D, Plotting the ratio of sucrose loss to the total labeled pool, we again observe a significant increase in RRP loaded when PMA is present during dye loading. ${ }^{* *} p<0.005$. Error bars indicate SE.

the increase in the amplitude of the fast component of release, suggesting that PMA application causes fusion of an additional set of vesicles rather than a simple increase in spontaneously recycling pool size (Fig. 5). Here, it is important to indicate that the spontaneously recycling vesicle pool size is readily saturable; even after prolonged dye application, only a limited number of vesicles can be labeled (Sara et al., 2005). However, the absence of a change in release kinetics when PMA treatment was performed after dye uptake strongly suggests that PMA does not act on spontaneously recycling vesicles after they have endocytosed.

\section{Folimycin treatment at rest causes depletion of} neurotransmitter from the RRP in the presence of PMA

In the next set of experiments, we used whole-cell recordings to test the premise that PMA causes spontaneous fusion of RRP vesicles. To test this prediction, we tagged spontaneously recycling vesicles by blocking neurotransmitter refilling after endocytosis at rest (Zhou et al., 2000) (Fig. 7A). Recordings obtained after 10 min incubation of hippocampal cultures with the vacuolar ATPase inhibitor folimycin in the presence of TTX revealed a sixfold reduction in mini frequency with only a small decrease in the amplitudes of individual spontaneous events (data not shown). Similarly, the decrease in the size of evoked synaptic current was $<20 \%$ after prolonged folimycin treatment (Fig. $7 B-$ $D$ ). These observations replicate the results of our previous study (Sara et al., 2005) and are consistent with the hypothesis that spontaneously fusing vesicles and vesicles fusing in response to presynaptic action potentials originate from distinct pools. Therefore, blocking vesicle refilling at rest (in TTX) selectively depletes neurotransmitter from spontaneously recycling vesicles, because these vesicles would lose their neurotransmitter during exoendocytosis. When we perfused hippocampal neurons with PMA along with TTX and folimycin for $10 \mathrm{~min}$, however, the evoked response amplitude detected after the treatment declined $>50 \%$ (Fig. $7 E-G$ ). This finding suggests that, in contrast to the treatment with folimycin plus TTX, the presence of PMA caused significant fusion from the RRP.

\section{Discussion}

Here, we studied the effect of the phorbol ester PMA on evoked and spontaneous synaptic vesicle recycling. In electrophysiological experiments, we found that the effect of PMA depended on maturational state of the synapses. Spontaneous neurotransmitter release from nascent synapses without a functional RRP was unresponsive to PMA application. In contrast, mature synapses responded robustly to PMA application, consistent with previous studies (Fig. 1). Mature synapses also substantially increased their responsiveness to action potential stimulation as well as hypertonic sucrose stimulation after PMA application (Fig. 2). This developmental specificity of the PMA action supports the premise that the PMA effect is specific to a set of vesicles resident in the RRP and does not affect vesicle fusion in general. Furthermore, the lack of a PMA effect on nascent synapse function suggests that this developmentally immature form of synaptic vesicle recycling is functionally similar to the spontaneous synaptic vesicle recycling detected in mature synapses.

In optical experiments, we observed that PMA increases the RRP size without any change in the size of the total recycling vesicle pool, regardless of whether PMA was applied before (Fig. 3), during, or after (Fig. 4) maximal FM dye uptake. The most striking effect was observed when the synaptic vesicles were preloaded with dye and then treated with PMA for 2 min after extracellular dye had been removed (Fig. 4A). The fact that, even in this case, there was an increase in the ratio of the sucrosereleasable RRP to the total pool strongly suggests that the effect of PMA is in redistributing the reserve pool vesicles into the RRP.

When we studied the effect of PMA during and after spontaneous dye uptake by vesicles (in the presence of TTX), we found that the presence of PMA during loading led to an increase in the size of the labeled vesicle pool (Fig. 5). This increase was accompanied by a switch from the normal slow monoexponential mode of dye release from spontaneously labeled vesicles (Sara et al., 2005) to a biexponential mode of release in response to $90 \mathrm{~mm}$ $\mathrm{K}^{+}$stimulation. We also detected a 10 -fold increase in the ability of sucrose to release dye when PMA was present during spontaneous loading compared with $4 \alpha$-PMA controls (Fig. 6). However, PMA treatment after spontaneous loading and dye washout did not result in any significant sucrose-induced release or a change in the kinetics of dye release. Thus, unlike in the case of activity-dependent dye uptake, PMA was unable to modify the distribution of vesicles that had been previously loaded with FM dye by spontaneous uptake. Together, these observations suggest that the additional vesicles mobilized by PMA belong to the activity-dependent recycling pool (presumably the RRP) rather than the spontaneously recycling pool.

Previous electrophysiological measurements showed that 
A

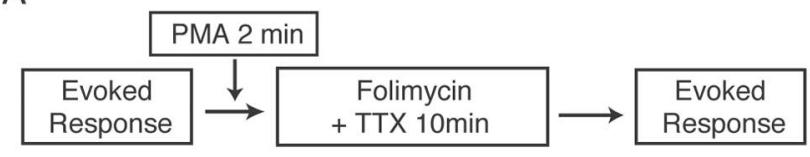

(-) PMA

$\mathrm{B}$

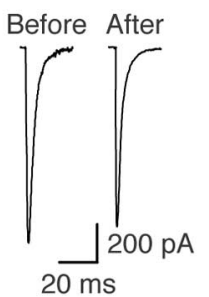
C

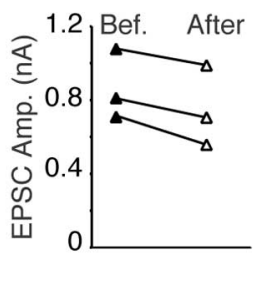

(+) PMA

E

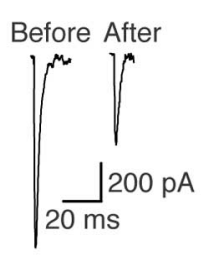

$\mathrm{F}$

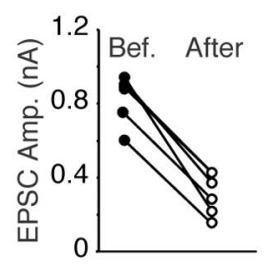

D

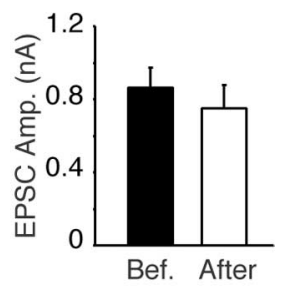

G

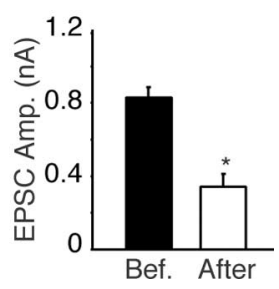

Figure 7. A, Neurons from 15 DIV cultures were electrically stimulated by local $200 \mu \mathrm{A}, 1 \mathrm{~ms}$ pulses and evoked responses were recorded electrophysiologically. Coverslips were treated with folimycin and TTX in between measurements of evoked response amplitudes. In another set of experiments, before folimycin and TTX treatment, cells were treated with $1 \mu \mathrm{m}$ PMA for 2 $\mathrm{min}$. After $10 \mathrm{~min}$ of treatment, evoked responses were recorded again. $\boldsymbol{B}$, Sample whole-cell patch-clamp recordings showing the evoked responses before and after folimycin treatment. $C$, Plot showing the change in EPSC amplitude by folimycin treatment in each individual experiment. $\boldsymbol{D}$, Bar graph showing no change in the evoked EPSC amplitudes during 10 min of folimycin treatment ( $n=3$ cells), replicating our previous results (Sara et al., 2005). E, Sample EPSC traces recorded from cells treated with both PMA and folimycin. $F$, Plots showing the change in amplitude of EPSCs in individual experiments before and after the combined treatment with PMA plus folimycin. G, Plot showing a significant decrease in the amplitudes of evoked responses in synapses treated with both PMA and folimycin $\left(n=5\right.$ cells). ${ }^{*} p<0.001$. Error bars indicate SE.

phorbol ester application increases the frequency of spontaneous miniature events. This finding may seem to conflict with the interpretation that PMA selectively acts on the activitydependent recycling pool of vesicles. However, our recent observation that the activity-dependent vesicle recycling and the spontaneous vesicle pool operate independently can help reconcile these apparently contradicting deductions (Sara et al., 2005). Spontaneous vesicle fusion and evoked vesicle fusion both occur at the active zone, and there does not appear to be any gross morphological difference in the size of the vesicles that give rise to the two forms of neurotransmission; thus, the release of single vesicles from these two distinct pools would be difficult to differentiate electrophysiologically. Nevertheless, in this study, we re-employed a strategy that we used previously to discern activity-dependent and spontaneously recycling vesicles using electrophysiology. Normally, treatment of cultures with blockers of vacuolar ATPase (such as folimycin) at rest causes selective depletion of neurotransmitter from the spontaneously recycling vesicles, because evoked response amplitudes are not significantly altered, and, after treatment, spontaneous event frequency diminishes substan-

tially (Sara et al., 2005) (Fig. 7). However, here, when we add PMA to the same protocol, we detected a significant reduction in the size of evoked response amplitudes (Fig. $7 E-G$ ). Thus, we propose that the apparent increase in spontaneous fusion by phorbol esters is mainly attributable to destabilization and fusion of RRP vesicles, presumably vesicles that have a high intrinsic $\mathrm{Ca}^{2+}$ sensitivity to begin with This premise is also consistent with the tendency for spontaneous dye loss from the activity-dependent recycling pool seen after PMA treatment (Fig. 4C). These vesicles may correspond to the highly $\mathrm{Ca}^{2+}$-sensitive vesicle pool seen in previous studies $(\mathrm{Wu}$ and $\mathrm{Wu}$, 2001; Yang et al., 2002).

What is the mechanism for this PMA effect? Traditionally, phorbol esters are thought to act primarily through the activation of PKC and subsequent PKC-dependent signaling cascades (Shapira et al., 1987; Stevens and Sullivan, 1998; Waters and Smith, 2000). However, recent evidence suggests that the presynaptic protein munc-13 is a major target for phorbol esters (as well as endogenous DAG) through its C1 domain (Betz et al., 1998). In genetically engineered mice that contain a mutated munc-13 with abolished phorbol ester binding, the PMA-induced increases in miniature-event fusion frequency and evoked transmission were abolished (Rhee et al., 2002). The results presented here are consistent with these observations. They also extend these findings by suggesting that phorbol esters act primarily through mobilization and restructuring of the activitydependent recycling pool. If munc-13 is located at the active zone, then how can its effect be selective to one vesicle versus the other? The simplest answer to this question would be that spontaneous vesicle fusion occurs outside the active zone. However, this assertion contradicts the finding that genetic deletion of all munc-13 homologs leads to complete cessation of neurotransmitter release, including spontaneous fusion (Varoqueaux et al., 2002). Therefore, one is left with the alternative explanation that vesicle priming requirements for spontaneous vesicle fusion are distinct from the reactions that prepare vesicles for activity-dependent fusion. In such a scenario, DAG signaling, as well as phorbol esters, may act at a late step in priming, which is not obligatory for spontaneous fusion.

\section{References}

Angleson JK, Betz WJ (2001) Intraterminal $\mathrm{Ca}^{2+}$ and spontaneous transmitter release at the frog neuromuscular junction. J Neurophysiol 85:287-294.

Berglund K, Midorikawa M, Tachibana M (2002) Increase in the pool size of releasable synaptic vesicles by the activation of protein kinase $\mathrm{C}$ in goldfish retinal bipolar cells. J Neurosci 22:4776-4785.

Betz A, Ashery U, Rickmann M, Augustin I, Neher E, Sudhof TC, Rettig J, Brose N (1998) Munc13-1 is a presynaptic phorbol ester receptor that enhances neurotransmitter release. Neuron 21:123-136.

Harata N, Pyle JL, Aravanis AM, Mozhayeva M, Kavalali ET, Tsien RW (2001) Limited numbers of recycling vesicles in small CNS nerve terminals: implications for neural signaling and vesicular cycling. Trends Neurosci 24:637-643.

Lonart G, Südhof TC (2000) Assembly of SNARE core complexes prior to neurotransmitter release sets the readily releasable pool of synaptic vesicles. J Biol Chem 275:27703-27707.

Malenka RC, Madison DV, Nicoll RA (1986) Potentiation of synaptic transmission in the hippocampus by phorbol esters. Nature 321:175-177.

Mozhayeva MG, Sara Y, Liu X, Kavalali ET (2002) Development of vesicle pools during maturation of hippocampal synapses. J Neurosci 22:654-665.

Rhee JS, Betz A, Pyott S, Reim K, Varoqueaux F, Augustin I, Hesse D, Sudhof TC, Takahashi M, Rosenmund C, Brose N (2002) Beta phorbol esterand diacylglycerol-induced augmentation of transmitter release is mediated by Munc13s and not by PKCs. Cell 108:121-133. 
Robinson DA, Zhuo M (2004) Modulation of presynaptic activity by phosphorylation in cultured rat spinal dorsal horn neurons. J Pain 5:329-337.

Rosenmund C, Stevens CF (1996) Definition of the readily releasable pool of vesicles at hippocampal synapses. Neuron 16:1197-1207.

Sara Y, Virmani T, Deak F, Liu X, Kavalali ET (2005) An isolated pool of vesicles recycles at rest and drives spontaneous neurotransmission. Neuron 45:563-573.

Shapira R, Silberberg SD, Ginsburg S, Rahamimoff R (1987) Activation of protein kinase $\mathrm{C}$ augments evoked transmitter release. Nature 325:58-60.

Stevens CF, Sullivan JM (1998) Regulation of the readily releasable vesicle pool by protein kinase C. Neuron 21:885-893.

Varoqueaux F, Sigler A, Rhee JS, Brose N, Enk C, Reim K, Rosenmund C (2002) Total arrest of spontaneous and evoked synaptic transmission but normal synaptogenesis in the absence of Munc13-mediated vesicle priming. Proc Natl Acad Sci USA 99:9037-9042.

Waters J, Smith SJ (2000) Phorbol esters potentiate evoked and spontaneous release by different presynaptic mechanisms. J Neurosci 20:7863-7870.

Wu XS, Wu LG (2001) Protein kinase C increases the apparent affinity of the release machinery to $\mathrm{Ca}^{2+}$ by enhancing the release machinery downstream of the $\mathrm{Ca}^{2+}$ sensor. J Neurosci 21:7928-7936.

Yang Y, Udayasankar S, Dunning J, Chen P, Gillis KD (2002) A highly $\mathrm{Ca}^{2+}$-sensitive pool of vesicles is regulated by protein kinase $\mathrm{C}$ in adrenal chromaffin cells. Proc Natl Acad Sci USA 99:17060-17065.

Zhou Q, Petersen CC, Nicoll RA (2000) Effects of reduced vesicular filling on synaptic transmission in rat hippocampal neurones. J Physiol (Lond) 525:195-206. 